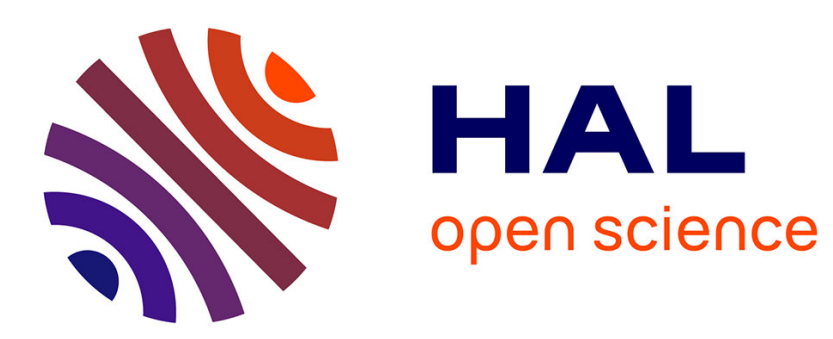

\title{
La réintroduction de l'Italie dans l'univers politico-culturel français après la Seconde Guerre mondiale: réseaux intellectuels et médiateurs culturels Olivier Forlin
}

\section{- To cite this version:}

Olivier Forlin. La réintroduction de l'Italie dans l'univers politico-culturel français après la Seconde Guerre mondiale: réseaux intellectuels et médiateurs culturels. Relations internationales, 2003, n. 116, p. 535-548. hal-01071609

\section{HAL Id: hal-01071609 \\ https://hal.univ-grenoble-alpes.fr/hal-01071609}

Submitted on 12 Oct 2015

HAL is a multi-disciplinary open access archive for the deposit and dissemination of scientific research documents, whether they are published or not. The documents may come from teaching and research institutions in France or abroad, or from public or private research centers.
L'archive ouverte pluridisciplinaire HAL, est destinée au dépôt et à la diffusion de documents scientifiques de niveau recherche, publiés ou non, émanant des établissements d'enseignement et de recherche français ou étrangers, des laboratoires publics ou privés.

\section{(ㅇ)(1) 80}

Distributed under a Creative Commons Attribution - NonCommercial - ShareAlikel 4.0 


\section{La réintroduction de l'Italie dans l'univers politico-culturel français après la Seconde Guerre mondiale : réseaux intellectuels et médiateurs culturels}

\section{Olivier Forlin}

La réinsertion de l'Italie dans les relations internationales, après la Seconde Guerre mondiale, est relativement rapide ${ }^{1}$. Amorcée dès 1947, après la signature du traité de paix, elle devient effective lorsque l'Italie adhère, en 1949, au pacte atlantique ; elle est confortée par la participation du pays, l'année suivante, aux premiers pas de la construction européenne, le projet de Communauté européenne de charbon acier (CECA). Dans le même temps, la France et l'Italie s'entendent pour rétablir des relations diplomatiques amicales dont témoigne le voyage de Georges Bidault à Turin en mars 1948 au cours duquel est annoncée l'intention des puissances anglo-saxonnes et de la France d'octroyer Trieste à l'Italie. Toujours lors de ce périple, un projet franco-italien d'union douanière est lancé ; certes, il ne verra pas le jour mais il constitue un bon indicateur de la nouvelle entente franco-italienne.

Ce processus s'explique essentiellement par le déclenchement de la guerre froide qui voit l'Italie, au regard de sa position géographique stratégique et de sa situation politique interne, devenir un enjeu majeur du conflit Est-Ouest ; les pays du bloc occidental cherchent désormais l'alliance italienne afin d'ancrer la péninsule dans le camp atlantique. Pareille évolution est par ailleurs permise et accélérée par la large victoire de la démocratie chrétienne de De Gasperi aux élections législatives d'avril 1948.

Le facteur culturel n'est toutefois pas totalement étranger à ce processus. La culture transalpine connaît en effet un important renouveau qui s'effectue sous le signe du néo-réalisme ${ }^{2}$. Plusieurs œuvres des deux principaux domaines concernés par ce renouvellement, la littérature et le cinéma, reçoivent un accueil enthousiaste en France au lendemain de la guerre ${ }^{3}$, particulièrement dans l'intelligentsia de gauche. L'engouement est le plus vif dans les milieux de la gauche marxiste indépendante du parti communiste, mouvance dont Les Temps Modernes et Esprit sont les revues les plus représentatives, ainsi que parmi des

1. Sur cette question, consulter, parmi les travaux de Pierre GuILLEN, « La réinsertion internationale de l'Italie après la chute du fascisme (1943-1947) », Relations Internationales, n 31, automne 1982, p. 333-349 ; «Les relations franco-italiennes de 1943 à 1949 », Revue d'Histoire diplomatique, janvier-juin 1976, p. 112-160.

2. Alberto ASOR RoSA, « La cultura », in Storia d'Italia, vol. IV : Dall'Unità a oggi, 2, Turin, Einaudi, 1975, p. 1604-1615.

3. Sur la réception du cinéma néo-réaliste en France, se reporter à l'article de Pierre MiLzA et Anne LE FUR, «L'accueil du cinéma italien en France au lendemain du second conflit mondial », in Jean-Baptiste DuROSELLE et Enrico SERRA (dir.), Il vincolo culturale fra Italia e Francia negli anni trenta e quaranta, Milano, ISPI/Franco Angeli, 1986, p. 304-329. 
groupes d'intellectuels membres du PCF, mais plutôt positionnés à la périphérie de la « citadelle» communiste. Séduits par le dynamisme retrouvé de la culture italienne, ces intellectuels sont également vivement attirés par les forces politiques qui, du PCI au parti d'action, en passant par le mouvement des catholiques communistes, le PSI et l'aile gauche de la démocratie chrétienne, animent la vie politique à gauche ${ }^{4}$. Ils s'intéressent, enfin, de près, aux débats idéologiques qui se développent autour des pensées d'Antonio Gramsci ${ }^{5}$ et d'Elio Vittorini, pour le pôle marxiste, et de celle de Piero Gobetti pour le pôle libéral de gauche. Or la réception et la mise en lumière de cette Italie-là sont à l'origine d'un discours nouveau sur ce pays, valorisant l'effervescence de la pensée et la créativité culturelle, et d'un profond renouvellement des images du peuple transalpin ${ }^{6}$.

Il est dès lors tentant d'établir un lien de cause à effet entre ces images positives et l'amélioration relativement rapide des relations diplomatiques franco-italiennes, accréditant ainsi l'idée d'un impact de ces représentations sur l'imaginaire des hommes politiques et des diplomates français. Aussi, le critique de cinéma Georges Sadoul n'hésitait-il pas à écrire dans la revue Europe en 1950 qu'un film comme Rome ville ouverte, de Roberto Rossellini, «montrant au monde la Résistance italienne a fait, pour que ce pays ne soit pas confondu avec le fascisme, plus que cent discours de cent ministres en exercice $»^{7}$. Il paraît toutefois difficile d'évaluer la portée exacte de représentations forgées essentiellement par des intellectuels de gauche : l'attrait pour la culture réaliste, les forces politiques de gauche et les débats idéologiques concerne presque exclusivement une mince élite culturelle.

Il est en possible en revanche de rechercher les origines et les fondements de ce discours et de ces images : il s'agit là d'analyser les modalités de la réintroduction et de la réception des réalités italiennes dans les milieux intellectuels français ; il convient aussi d'identifier les acteurs de la diffusion de la connaissance de l'Italie en France. Des processus dans lesquels interviennent des médiateurs culturels franco-italiens et des réseaux intellectuels qui ont une fonction déterminante de passeurs culturels ; ils conservent également une influence sur les

4. L'attitude des intellectuels des Temps Modernes et d'Esprit à l'égard de l'Italie après la guerre a été étudiée par Pierre MiLZA, «L'Italie de l'après-guerre vue par deux organes de la "nouvelle gauche": Les Temps Modernes et Esprit », in Jean-Baptiste DUROSELLE et Enrico SERRA (dir.), Italia e Francia (1946-1954), Milano, ISPI/Franco Angeli, 1988, p. 185-204.

5. Sur la réception de Gramsci en France au lendemain de la guerre, voir Olivier FoRLIN, « Un mythe au service de combats politiques. L'image de Gramsci chez les intellectuels français de gauche de 1945 à 1948 », in Gilles BERTRAND (dir.), Identité et cultures dans les mondes alpin et italien, XVIII-XX siècles, Paris, L'Harmattan, 2000, p. $165-206$.

6. Pierre Milza et Anne Le Fur soulignent en effet qu'à partir des films néo-réalistes sont mises en évidence l'idée d'une dissociation des Italiens et du régime fasciste, et les représentations d'un peuple qui, face aux épreuves de la guerre, de la résistance et de la misère sociale, a su se montrer « grave, digne, courageux [...] et profondément humain » (article cité, p. 322-329, citation p. 326).

7. Georges Sadoul, « Le néo-réalisme italien », Europe, n ${ }^{52}$, avril 1950, p. 131. 
réalités qu'ils transmettent puisqu'ils effectuent des choix, des interprétations et marquent ainsi de leur empreinte les faits propagés. Aussi est-il indispensable de connaître ces médiateurs leurs itinéraires et l'origine de leur italophilie - et ces réseaux - les modalités de leur constitution, leur fonctionnement -, afin de saisir comment s'est effectuée la réintroduction de l'Italie et les conditions de sa réception dans l'univers politico-culturel français.

Trois facteurs paraissent déterminants pour répondre à ces interrogations : le premier est historico-politique et renvoie au rôle de l'émigration politique italienne dans l'entre-deux-guerres, tandis que les deux autres sont d'ordre culturel et ont trait, l'un à la sphère éditoriale, l'autre aux réseaux intellectuels liés à la revue Esprit et au mouvement personnaliste.

Le facteur historico-politique : les relations entre émigrés politiques italiens et intellectuels français

L'émigration italienne antifasciste a une fonction importante dans le processus de réintroduction de l'Italie dans la France du second après-guerre, à la fois directe et, plus encore, indirecte.

Rôle direct tout d'abord puisque certains médiateurs culturels qui font connaître les réalités transalpines en France à partir de 1945 sont des Italiens d'origine. Issus de l'émigration antifasciste de l'entre-deux-guerres, ils interviennent dans les revues et hebdomadaires français à travers des articles présentant et étudiant la culture, les courants idéologiques et les partis politiques italiens. Le parcours de Maria Brandon-Albini est une bonne illustration de ce phénomène ${ }^{8}$. Née en 1904 en Lombardie, installée avec sa famille à Milan au cours de la Première Guerre mondiale, elle participe à une opposition de type culturel au fascisme menée par des écrivains et artistes milanais au moyen de petites revues et de diverses réunions. En 1936 elle choisit l'exil à Paris où elle fréquente les milieux de l'émigration antifasciste, communistes notamment. Ainsi collabore-t-elle à La Voce degli Italiani, le quotidien de l'Union populaire italienne, organisation antifasciste que contrôlent les communistes ${ }^{9}$. Elle noue également des relations dans les milieux intellectuels français, publie des textes dans Europe et rencontre Pierre Brandon, un avocat engagé dans les rangs du PCF. À l'été 1940, le couple rejoint la zone libre et participe à la Résistance communiste à Toulouse, puis Marseille et Nice. À la faveur de son mariage, Maria Brandon-Albini choisit de rester en France à la

8. Elle a laissé des mémoires : Maria BRANDON-Albini, De la terre de Lombardie à Montmartre, Paris, Éd. Entente, 1988.

9. Éric VIAL, « L'Unione Popolare Italiana (UPI), 1937-1940. Une organisation de masse communiste en exil », $L a$ Trace, $\mathrm{n}^{\circ} 11-12$, mai 1999, p. 11-34. 
Libération. Compagnon de route du parti communiste, intégrée dans les milieux intellectuels du parti, elle collabore à divers périodiques liés au PCF, Europe, Action ou encore Arts de France, où, forte de son expérience et de son expertise, elle est en mesure d'analyser l'évolution de la culture transalpine depuis le début du $\mathrm{XX}^{\mathrm{e}}$ siècle, les itinéraires des intellectuels italiens sous le fascisme et la guerre, et les nouveaux courants et auteurs qui animent l'univers culturel du second après-guerre ${ }^{10}$.

Lui aussi exilé en France au milieu des années trente, Nicola Chiaromonte, militant du mouvement fondé et dirigé par Carlo Rosselli, Giustizia e Libertà $(G L)$, rejoint les États-Unis pendant le conflit mondial. C'est depuis l'Italie, retrouvée après la guerre, qu'il rédige des textes publiés dans les colonnes de revues françaises, Les Temps Modernes au cours de la seconde moitié des années quarante, puis Preuves dans la décennie suivante ${ }^{11}$ : Chiaromonte est en effet, avec l'homme politique et écrivain Ignazio Silone, l'un des principaux animateurs en Italie du Congrès pour la liberté de la culture ; les deux intellectuels lancent et dirigent en 1955 la revue Tempo presente, l'équivalent transalpin de Preuves $^{12}$.

Les exemples de Maria Brandon-Albini et Nicola Chiaromonte demeurent toutefois isolés et les anciens fuorusciti sont assez peu nombreux à intervenir dans les périodiques français pour faire connaître les réalités italiennes : la plupart d'entre eux sont en effet rentrés en Italie dès juillet 1943 pour participer à la Résistance et à la reconstruction de leur pays ${ }^{13}$.

L'émigration politique italienne a cependant eu un rôle indirect significatif dans la réintroduction de l'Italie en France, qui se manifeste par une influence sur un groupe de médiateurs culturels, des Français d'origine, qui ont été en contact avec des fuorusciti dans les années trente et durant la guerre. Un groupe assez homogène politiquement et du point de vue générationnel. Nés entre 1914 et 1921, éveillés à la conscience politique sous le signe de la lutte antifasciste à partir du milieu des années 1930, ses membres forment une génération intellectuelle. Leurs engagements et leurs parcours politiques présentent de nombreuses similitudes : ils adhèrent au PCF au cours des années trente, lorsque le parti est, après le tournant stratégique de 1934, à la pointe du rassemblement de la gauche contre le danger fasciste, participent à la Résistance, maintiennent pour la plupart leur fidélité au parti

10. Parmi ses articles, on peut citer, à titre d'exemples, Maria Brandon-Albini, «La résistance dans la littérature italienne (1922-1944) », Action, $\mathrm{n}^{\circ} 78,1^{\mathrm{er}}$ mars 1946, p. 12-13; «Italie. Les nouvelles orientations du roman italien contemporain », Europe, n ${ }^{\circ} 23$, novembre 1947, p. 135-141 ; « Nouvelles orientations de la peinture italienne », Arts de France, ${ }^{\circ}$ 11-12, 1947, p. 57-65.

11. Nicola Chiaromonte, «Chronique italienne», Les Temps Modernes, $n^{\circ} 38$, novembre 1948, p. 930-943 ; «Lettre d'Italie », Preuves, ${ }^{\circ}$ 11, janvier 1952, p. 27-31.

12. Le rôle de Silone et Chiaromonte dans le Congrès pour la liberté de la culture a été analysé par Pierre Gremion, Intelligence de l'anticommunisme. Le Congrès pour la liberté de la culture à Paris, 1950-1975, Paris, Fayard, 1995 , p. 55-58 et 401-402.

13. Éric ViaL, « Notes sur le retour des émigrés politiques de France en Italie», in Gianni Perona (dir.), Gli Italiani in Francia, 1938-1946, Mezzosecolo, n 9, 1993, p. 59-76. 
communiste dans l'après-guerre tout en se situant aux marges de l'organisation ; les habitudes d'indépendance, ou tout au moins de distance, par rapport aux autorités, héritées de la clandestinité, l'empreinte de l'adhésion à un communisme conçu comme ouvert et rassembleur, et l'attachement à une relative liberté culturelle, expliquent ce positionnement périphérique. Quelques-uns, enfin, demeurent indépendants du PCF depuis leur rupture avec lui avant le second conflit mondial ; se proclamant communistes antistaliniens, ils militent dans la mouvance trotskiste.

Parmi les principaux représentants de cette génération intellectuelle devenus des médiateurs franco-italiens figurent Jean-Pierre Vernant (né en 1914), Dionys Mascolo (1916), Dominique Desanti (1919), Edgar Morin (1921) ; membres du PCF, ils collaborent à l'hebdomadaire Action où ils constituent le courant le plus ouvert ${ }^{14}$; Mascolo et Edgar Morin forment, avec Marguerite Duras (1914) et Robert Antelme (1917), le groupe de la rue Saint-Benoît. Avec Dominique Desanti ${ }^{15}$, on peut également citer son mari, Jean-Toussaint Desanti (1914) et ses camarades d'études à la khâgne de Lakanal, Pierre Hervé (1914) et Pierre Courtade (1915), tous deux membres du comité de direction d'Action. Gilles Martinet, né en 1916, a quant à lui quitté le PCF en 1938 à la suite de son opposition à la condamnation de Boukharine ; il participe au lendemain de la guerre à La Revue internationale qui rassemble des intellectuels trotskistes (notamment Pierre Naville, le directeur du périodique) ou proches de cette mouvance ${ }^{16}$. Ces intellectuels ont pour dénominateur commun d'avoir connu des exilés politiques italiens avant et/ou pendant la guerre et d'avoir été particulièrement sensibles à la propagande développée par certains courants du fuoruscitismo - les groupes communistes et giellistes furent les plus actifs en ce domaine ${ }^{17}$-, parce qu'ils étaient en train de s'éveiller, à ce moment-là, à la politique sous la forme de la mobilisation antifasciste. Ces liens ont déterminé chez eux un intérêt pour l'Italie les conduisant à effectuer des périples outre-monts après la guerre, à rétablir des relations avec les anciens exilés et, s'informant auprès d'eux, à faire connaître les réalités de l'Italie nouvelle en France. La génération intellectuelle antifasciste est une génération italophile.

14. Les orientations d'Action sont analysées par Jeannine Verdes-Leroux, Au service du Parti. Le parti communiste, les intellectuels et la culture (1944-1956), Paris, Fayard/Ed. de Minuit, 1983, p. 197-203.

15. Se reporter à ses mémoires Les Staliniens. Une expérience politique (1944-1956), Paris, Fayard, 1975, et Ce que le siècle m'a dit, Mémoires, Paris, Plon, 1997, p. 57-58, et 259-269 notamment.

16. Gilles MARTINET, Cassandre et les tueurs. Cinquante ans d'une histoire française, Paris, Grasset, 1986, p. 62-69.

17. Pierre GuILLEN, « Le rôle politique de l'immigration italienne en France dans l'entre-deux-guerres », in Pierre MiLZA (dir.), Les Italiens en France de 1914 à 1940, Rome, École française de Rome, 1986, p. 323-341 ; Idem, «La risonanza in Francia dell'azione di GL e dell'assassinio dei fratelli Rosselli », in Giustizia e Libertà nella lotta antifascista e nella storia d'Italia, Florence, La Nuova Italia, 1978. 
L'exemple le plus significatif est celui de Gilles Martinet dont l'intérêt pour l'Italie a pour origine sa rencontre avec la fille cadette du responsable syndical en exil Bruno Buozzi. Il peut dès lors connaître plusieurs chefs de file de l'antifascisme transalpin réfugiés en France, notamment des socialistes dont son beau-père était proche (entre autres Pietro Nenni et Giuseppe Saragat). Gilles Martinet multiplie les séjours en Italie dès la fin de la guerre, renoue le contact avec les anciens fuorusciti, tisse également des liens nouveaux parmi les hommes politiques et les intellectuels de gauche, et peut, par ces réseaux de relations, jouer un rôle de médiateur culturel ; l'information sur la péninsule, dans les périodiques auxquels il collabore, passe par lui (à La Revue internationale d'abord, puis à France-Observateur dont il est l'un des fondateurs $)^{18}$. Autre indice témoignant de l'existence d'une relation entre l'émigration politique italienne et l'italophilie de certains intellectuels français : Jean-Pierre Vernant, Dominique Desanti, Edgar Morin et Gilles Martinet ont tous connu pendant la guerre ${ }^{19}$, ou été en contact avec lui ${ }^{20}$, Silvio Trentin, l'un des responsables de $G L$; réfugié en France en 1926, installé à Toulouse depuis 1934, il avait ouvert là une librairie et fondé, en 1941, le mouvement de résistance Libérer et Fédérer ainsi qu'une revue homonyme dont l'audience était importante dans la région toulousaine ${ }^{21}$. S'ils ne le revoient pas - Trentin mourut en Italie en 1944 -, Jean-Pierre Vernant et Edgar Morin nouent des contacts après la guerre avec des militants du parti d'action, l'héritier de $G L$; J.-P. Vernant affirme avoir voulu les rencontrer parce que certains d'entre eux avaient combattu à ses côtés, pendant la Résistance, dans le Sud-Ouest ${ }^{22}$. Par ailleurs, René Maheu, camarade d'études de Sartre en khâgne et à l'ENS d'Ulm, originaire de Saint-Gaudens, connut Trentin à Toulouse où il fit ses études secondaires ; en 1946, il dédicace l'article sur l'Italie nouvelle qu'il publie dans Les Temps Modernes «au regretté Silvio Trentin $»^{23}$.

Les contacts entre exilés italiens et intellectuels français permettent de saisir les origines de l'attrait pour l'Italie d'une génération intellectuelle qui exerce sa fonction médiatrice à partir de 1945. Toutefois, ce facteur historique conduit à cerner seulement l'amorce de l'italophilie de

18. Entretien avec Gilles Martinet, du 22 décembre 1997. Voir aussi son essai, Les Italiens, Paris, Grasset, 1990. 19. Entretiens avec Jean-Pierre Vernant, du 14 décembre 1997, Dominique Desanti, du 8 décembre 1997, et Edgar Morin, du $1^{\text {er }}$ octobre 1998.

20. C'est le cas de Gilles Martinet dont le groupe de résistance auquel il participait à Clermont-Ferrand fut en relation avec Trentin et son mouvement toulousain. Archives Gilles Martinet, CHEVS/FNSP, MR 1 : La Presse, 1938-1948, Dossier 2 : Coupures de presse, notes, littérature, 1944.

21. Consulter sur ce point l'ouvrage collectif Silvio Trentin e la Francia. Saggi e testimonianze, Venise, Marsilio, 1991, en particulier l'article de H. W. TOBLER, «Silvio Trentin et son influence idéologique sur les idées politiques et sociales de la Résistance française », p. 99-107.

22. C'est le cas, en particulier, de Mario Levi. Entretien avec J.-P. Vernant, du 14 décembre 1997.

23. René Maheu, «Italie nouvelle ou les incertitudes de la liberté », Les Temps Modernes, $\mathrm{n}^{\circ}$ 10, juillet 1946, p. 64. Sur Maheu, se reporter à la thèse de Jean-François Sirinelli, Génération intellectuelle. Khâgneux et Normaliens dans l'entre-deux-guerres, Paris, PUF, 1994, p. 362-364. 
certains clercs et les prémices de la reprise des relations intellectuelles franco-italiennes. Car si les premières relations sont rétablies parmi les anciens exilés, rapidement ensuite des intellectuels français tissent des liens avec des clercs italiens qui étaient restés en Italie pendant le fascisme, ce qui fut le cas de la plupart des écrivains et des artistes ${ }^{24}$. D'autres facteurs, qu'il convient d'examiner, sont donc à l'œuvre et prennent le relais dans le processus de réintroduction de l'Italie en France.

\section{Les facteurs culturels : le rôle de la sphère éditoriale}

C'est à travers la diffusion et la réception de l'œuvre de l'écrivain Elio Vittorini que le rôle de la sphère éditoriale est le plus manifeste. Quoique divers, les médiateurs culturels et les réseaux intellectuels qui accueillent et font connaître ses romans et ses réflexions partagent cependant un dénominateur commun : leurs liens avec les éditions Gallimard.

Né en 1908 en Sicile, resté en Italie sous le régime fasciste, Elio Vittorini passe progressivement à l'antifascisme culturel à la fin des années trente avant de s'engager dans la Résistance milanaise en 1943-1945 et d'adhérer au PCI. Il effectue dès la fin 1945, puis au début du printemps 1946, des voyages en France où il peut s'insérer dans deux réseaux de l'intelligentsia. Celui des clercs du PCF, en premier lieu, avec lequel il entre en contact lorsqu'il est reçu, entre autres par Aragon, au Comité national des Écrivains (CNE). Selon un témoignage de Dionys Mascolo, il se sent toutefois mal à l'aise en raison de l'atmosphère empreinte de rigidité régnant au $\mathrm{CNE}^{25}$. Claude Roy, enthousiasmé par ses romans ${ }^{26}$, l'emmène alors rue Saint-Benoît où il peut côtoyer un milieu plus conforme à ses attentes et où il tisse de solides liens d'amitié : Dionys Mascolo, Marguerite Duras, Robert Antelme et Edgar Morin, ainsi que Claude Roy, deviennent ses proches, comme en témoignent les lettres qu'il échange avec eux au cours des années suivantes ${ }^{27}$. Vittorini noue également des relations avec des membres de l'équipe des Temps Modernes qu'il voit à Paris au début de 1946. Peu de temps

24. Pierre Milza, «Cultures et pratiques culturelles », in Pierre Milza et Denis Peschanski (dir.), Exils et migration. Italiens et Espagnols en France, 1938-1946, Paris, L'Harmattan, 1994, p. 324-325.

25. Vittorini a alors affirmé se sentir « en cage » (témoignage de Dionys Mascolo cité dans « Une vie, une œuvre : Elio Vittorini », émission réalisée par Francesca Isidori et diffusée sur France-Culture en juin 2000).

26. Il affirme en effet dans ses mémoires qu'il lut des romans de Vittorini, édités en Belgique et en Suisse avant de l'être en France, dès 1944. De ces lectures vint son désir de connaître l'écrivain, à qui il consacre un chapitre de ses mémoires, puis son intérêt pour l'Italie (Claude RoY, Nous, Paris, Gallimard, 1972, p. 195-215).

27. La correspondance de Vittorini des années 1945-1951 a été éditée : E. Vittorini, Gli anni del «Politecnico ». Lettere, 1945-1951, Turin, Einaudi, 1977. Les cinq intellectuels sont invités à plusieurs reprises par Vittorini à Milan et dans sa résidence d'été. C'est surtout avec D. Mascolo et C. Roy que Vittorini correspond (voir, par exemple, les lettres d'E. Vittorini à C. Roy, du 20 décembre 1947, du 18 mars 1948 et du 31 mars 1949, in Gli anni..., op. cit., p. 147-148, 157-160 et 241-242 ; et celles adressées à D. Mascolo, du $1^{\text {er }}$ juin 1948, du 15 juin 1949, etc., in op. cit., p. 170-173 et 258-260). 
après, Jean-Paul Sartre et Simone de Beauvoir effectuent un périple en Italie au cours duquel ils sont accueillis à Milan par l'écrivain transalpin et des membres du Politecnico, le périodique qu'il dirige $^{28}$. C'est au cours de ce voyage que Sartre a l'idée de consacrer un substantiel numéro spécial des Temps Modernes à l'Italie ; publié en août-septembre 1947, ce volume constitue une étape importante de la connaissance de l'Italie en France ${ }^{29}$. Or, il doit beaucoup à Vittorini qui s'est chargé de rassembler des articles rédigés par des collaborateurs du Politecnico (Stefano Terra et Franco Fortini en particulier) ou d'autres intellectuels parmi ses proches (notamment des membres, tel Remo Cantoni, de la revue milanaise Studi filosofici, ou, comme Romano Bilenchi ${ }^{30}$, de Società, périodique florentin) ; des textes inédits ou parus précédemment dans les colonnes de revues italiennes ${ }^{31}$. Vittorini a donc eu ici une fonction de médiateur culturel, contribuant à diffuser la connaissance de l'Italie en France.

Dans le même temps, il est aussi l'objet de la médiation puisque ses propres œuvres parviennent en France. Les Temps Modernes font en effet paraître plusieurs extraits de l'un de ses romans avant son édition française ${ }^{32}$. Ses livres sont progressivement traduits et publiés et, indice de sa notoriété croissante, le délai de traduction de ses romans est de plus en plus réduit puisque si des livres comme Petite bourgeoisie (publié en 1931 en Italie) et L'Eillet rouge (1934) ne sont traduits, respectivement, que dix-sept et seize ans plus tard, et si Conversation en Sicile (1941) doit encore attendre sept ans avant de paraître en français, le délai de traduction des Hommes et les autres tombe à deux ans seulement (1945-1947) et celui du Simplon fait un clin d'œil au Fréjus à trois ans (1947-1950) ${ }^{33}$; on peut noter par ailleurs que ces cinq romans paraissent dans un laps de temps relativement court, les années 1947-1950.

C'est Gallimard qui a un rôle essentiel dans cette entreprise éditoriale, car à l'exception de Petite bourgeoisie ${ }^{34}$, toutes les œuvres de Vittorini sont publiées par la prestigieuse maison et traduites par Michel Arnaud qui travaille principalement pour elle. Très lié à Vittorini, il est

28. Simone de Beauvoir évoque ce voyage dans ses mémoires, La force des choses, vol. I, Paris, Gallimard (coll. Folio), 1990 (1 $1^{\text {ère }}$ éd. 1963), p. 136-138.

29. Italie, Les Temps Modernes, $\mathrm{n}^{\circ}$ 23-24, août-septembre 1947.

30. Vittorini lui a demandé de donner un texte, précédemment publié dans Società, pour le numéro italien des Temps Modernes (lettres d'E. Vittorini à R. Bilenchi, du 15 janvier 1947 et du 29 janvier 1947, in E. Vittorini, op. cit., p. 100 et 105).

31. L'équipe des Temps Modernes n'a toutefois pas totalement repris le projet élaboré par Vittorini qui est reproduit dans Gli anni..., op. cit., p. 419-420. Sartre a en effet fait appel à des intellectuels issus d'autres milieux, notamment à Carlo Levi, membre du parti d'action (lettre d'Elio Vittorini à Carlo Levi, du 21 septembre 1946 ; lettre d'Elio Vittorini à Massimo Mila, du 25 mars 1947, in E. Vittorini, op. cit., p. 71 et 114). Carlo Levi a très certainement rassemblé les textes d'Aldo Garosci et de Manlio Rossi Doria, tout deux également membres du parti d'action.

32. Elio Vittorini, Le Simplon fait un clin d'œil au Fréjus, Les Temps Modernes, n 19, avril 1947, p. 1153-1193, et $\mathrm{n}^{\circ} 20$, mai 1947, p. 1376-1409.

33. Se reporter sur ce point aux travaux de Jean-Pierre VIALLET, «Le livre, témoin des relations culturelles entre l'Italie et la France (1945-1958) », MEFRM, 98, 1986-1, p. 465-524.

34. Le roman est traduit par Maria Brandon-Albini et édité en 1948 par la maison Marin. 
son traducteur quasi exclusif ${ }^{35}$. Il est également proche de l'équipe des Temps Modernes pour laquelle il a traduit plusieurs des textes du numéro italien de 1947 ; or la revue dirigée par Sartre est éditée, jusqu'à la fin 1948, par Gallimard. Michel Arnaud est enfin lié aux intellectuels de la rue Saint-Benoît, particulièrement à Dionys Mascolo qui, à ce moment-là, travaille comme lui chez Gallimard $^{36}$. La réception et la diffusion des œuvres de Vittorini en France passent par l'intermédiaire de quelques médiateurs culturels et de deux réseaux intellectuels auxquels l'écrivain s'est agrégé à la faveur de ses séjours en France. L'un de ses réseaux, celui structuré autour des Temps Modernes, est influent dans la cléricature française et possède un fort pouvoir de légitimation, permettant à Vittorini d'obtenir une reconnaissance significative dans l'intelligentsia ; reconnaissance que le prestige des éditions Gallimard, qui constituent le point de jonction entre les divers acteurs introduisant l'œuvre de l'écrivain transalpin, vient conforter.

\section{Les facteurs culturels : les réseaux intellectuels liés à la revue Esprit}

D’autres réseaux mettant en relation des intellectuels français et italiens se constituent dans l'immédiat après-guerre. Ils dépendent de la revue Esprit et lui sont spécifiques.

Esprit est en effet une revue autour de laquelle s'est formé depuis les années trente un mouvement qui a donné naissance, en France et à l'étranger, à des centres personnalistes. Certes, après la guerre, le mouvement perd de son dynamisme ${ }^{37}$. Il ne disparaît cependant pas et des intellectuels italiens se préoccupent de créer des centres qui lui sont affiliés et, pour cela, entrent en contact avec Emmanuel Mounier. Les premiers à le faire appartiennent tous à la nébuleuse des chrétiens de gauche. Les liens les plus étroits noués par le directeur d'Esprit avec des intellectuels transalpins le sont avec des catholiques communistes, un courant qui, emmené par Franco Rodano, a lancé pendant la Résistance la Sinistra cristiana $(S C)$. La formation s'est dissoute peu après le conflit, en 1946, à la suite du désaveu du Vatican et la plupart de ses membres sont allés rejoindre les rangs du PCI. Parmi les catholiques communistes, le groupe le plus important pour Esprit est celui qui se réunit à Turin autour de Felice Balbo, l'un des théoriciens de la $S C$, et du philosophe Augusto del Noce. La correspondance entre Mounier et Balbo commence en février 1946 et le centre personnaliste turinois est lancé à la fin de la même année. À Milan se réunit un autre groupe de sensibilité analogue sous l'égide d'Alessandro Pellegrini qui fonde un centre au milieu de 1947 ; ce dernier s'apparente toutefois davantage à

35. Seize lettres de l'écrivain adressées à Michel Arnaud figurent dans Gli anni..., op. cit..

36. Voir sur ce point les lettres d'E. Vittorini à M. Arnaud, du 21 décembre 1946, du 7 juillet 1947, du 11 novembre 1947 et de février-mars 1948, in E. Vittorini, op. cit., p. 96-97, 124, 145-146 et 155-156.

37. Michel WinOcK, «Esprit». Des intellectuels dans la cité, 1930-1950, Paris, Le Seuil, 1996 (1 ${ }^{\text {ère }}$ éd. 1975), p. 256-257. 
un cercle intellectuel et culturel et paraît moins strictement affilié au mouvement Esprit que ne l'est son homologue turinois ${ }^{38}$. Des liens étroits sont également tissés avec des intellectuels du mouvement Comunità constitué à l'initiative du chef d'entreprise mécène Adriano Olivetti et basé à Ivrée. Tout en se réclamant de la pensée de Mounier, ce groupe est lui aussi plus distant que le centre turinois par rapport au mouvement personnaliste ; il est d'orientation chrétienne et socialisante plus que communisante. Toujours dans la mouvance de la gauche chrétienne, des contacts sont établis avec des représentants du courant des Cronache sociali, l'aile gauche de la démocratie chrétienne, qui dispose d'une revue homonyme à partir de 1947. C'est avec le directeur du périodique, Giuseppe Glisenti, et non avec les ténors du courant - Giuseppe Dossetti, Amintore Fanfani et Giorgio La Pira -, que Mounier entretient une relation épistolaire $^{39}$.

Ces réseaux d'intellectuels ont une fonction de premier ordre quant aux relations entre Esprit et l'Italie, à l'instar du groupe formé par Balbo et ses amis turinois. Ces derniers sont, en premier lieu, demandeurs vis-à-vis du directeur d'Esprit, comme en témoignent les requêtes qu'ils formulent dans une série de missives échangées entre février et octobre 1946. Felice Balbo demande ainsi à Mounier de lui faire parvenir ses principaux textes sur le personnalisme - Situation du personnalisme, puis le Manifeste du personnalisme ${ }^{40}$-, lui propose de les traduire et de les éditer en Italie - il travaille pour l'éditeur Einaudi ${ }^{41}$; il lui demande de lui envoyer tous les numéros d'Esprit parus depuis la fin 1944 et se réclame explicitement de la pensée de son correspondant ${ }^{42}$; il sollicite également ses conseils pour la création du centre personnaliste turinois dont il ressent « un grand besoin ${ }^{43}$ et qui voit donc le jour fin 1946. Emmanuel Mounier ne reste toutefois pas passif à l'égard de ces initiatives, et ce pour deux raisons. C'est en effet dans le mouvement de la Sinistra cristiana et sa filiation qu'il voit le noyau d'un personnalisme italien marxiste ${ }^{44}$; aussi accueille-t-il favorablement toutes les requêtes de Balbo et de son groupe, leur envoyant textes, numéros de sa revue, acceptant leurs

\footnotetext{
38. Voici en effet ce que Pellegrini affirme à Mounier : « Je me suis occupé de former à Milan et à Rome des groupes qui soient orientés selon l'inspiration de votre mouvement, mais en pleine liberté d'évaluation »; lettre d'Alessandro Pellegrini à E. Mounier, du 11 mars 1947, Archives Esprit, ESP2 C1 02 02: «Correspondance générale avec l'Italie (1946-1953) », Institut Mémoires de l'Édition contemporaine (IMEC). Le groupe romain évoqué dans cette lettre ne verra pas le jour.

39. G. Glisenti adresse une lettre au directeur d'Esprit afin de lui présenter la revue qu'il dirige, lancée quelques mois plus tôt (lettre de G. Glisenti à E. Mounier, du 28 octobre 1947, réf. citées).

40. Lettres de F. Balbo à E. Mounier, du 15 février 1946, et du 2 mai 1946, réf. citées.

41. Lettres de F. Balbo à E. Mounier, du 2 mai 1946 et du 12 février 1948, réf. citées.

42. Voici ce qu'il écrit à Mounier : " Quand j'ai lu Situation du Personnalisme, j'ai senti une consonance entre mes recherches et mes pensées et votre production culturelle et politique » (lettre de F. Balbo à E. Mounier, du 2 mai 1946, réf. citées).

43. Ibid.

44. Pierre MILZA, «L'Italie de l'après-guerre vue par deux organes de la "nouvelle gauche" : Les Temps Modernes et Esprit », article cité, p. 200.
} 
propositions d'édition, et tient-il en estime ses interlocuteurs ${ }^{45}$. Corrélativement, on est, en 1945-1947, à une période où Mounier cherche à établir des liens, qu'il n'a pas, avec des groupes d'intellectuels transalpins et, plus généralement, avec la culture italienne. Dans cet état d'esprit, il sollicite, en avril 1946, Felice Balbo pour qu'il lui indique «les principaux milieux avec lesquels il serait intéressant d'être en relation en Italie et les revues qui le méritent ${ }^{46}$; quelques mois plus tard, il demande à son correspondant de lui faire parvenir « des œuvres doctrinales ou littéraires susceptibles et dignes d'être traduites ${ }^{47}$. Le philosophe français entretient le même type de relations avec Adriano Olivetti, avec qui il entre précocement en contact, au même moment qu'avec Balbo en février 1946, et Alessandro Pellegrini.

Ces réseaux d'intellectuels ont ainsi un rôle actif de médiateurs : à la fois pour faire connaître aux lecteurs d'Esprit la culture et les idéologies italiennes, et pour introduire Mounier et les siens dans d'autres réseaux intellectuels et culturels, l'autoriser à nouer des relations plus amples. Ce sont en effet Balbo, Olivetti et Pellegrini qui se chargent de l'organisation de la tournée de conférences que Mounier effectue en Italie à la fin de 1947 ; au cours de ce périple qui le conduit d'Ivrée à Rome en passant par Turin, Milan et Florence, ses trois hôtes lui font rencontrer, conformément à ses espérances ${ }^{48}$, les représentants d'autres milieux de l'intelligentsia italienne : des communistes, en particulier ceux qui animent à Milan, autour de Vittorini, Il Politecnico, les rédacteurs (entre autres Antonio Banfi, son directeur, et Remo Cantoni, son rédacteur en chef) de Studi filosofici, ceux qui gravitent autour de la Casa della cultura $^{49}$ de la ville et, à Florence, les responsables de la revue Società ${ }^{50}$; des intellectuels membres ou héritiers du parti d'action, notamment l'équipe d'Il Ponte que dirige à Florence Piero Calamandrei ${ }^{51}$.

À son retour d'Italie, Mounier fait paraître dans Esprit une série d'articles consacrés à la péninsule. Dans le premier, qu'il signe, sont présentés les principaux milieux intellectuels qu'ils a rencontrés, leurs débats, ainsi que les forces politiques de gauche ${ }^{52}$; ce texte est suivi

45. Lettres d'E. Mounier à F. Balbo, du 15 avril 1946 et du 7 octobre 1946, réf. citées.

46. Lettre d'E. Mounier à F. Balbo, du 15 avril 1946, réf. citées.

47. Lettre d'E. Mounier à F. Balbo, du 7 octobre 1946, réf. citées.

48. Il confie à Pellegrini que « Les conférences sont un moyen d'assurer matériellement mon voyage et de prendre contact avec un vaste public. Mon but essentiel est de rencontrer, en dehors de tous les milieux officiels, les éléments vivants de la jeune Italie, et j'insiste sur ce point car je sais par expérience combien ces libres contacts sont plus féconds que les déjeuners officiels et les réunions de foule » (lettre d'E. Mounier à A. Pellegrini, du 22 mai 1947, réf. citées).

49. Ce sont Balbo (lettre de F. Balbo à E. Mounier, du 29 août 1947, réf. citées) et Olivetti (lettre d'A. Olivetti à E. Mounier, du 10 octobre 1947, réf. citées) qui indiquent à Mounier la Maison de la culture milanaise dont les responsables sont liés au PCI.

50. Lettre de F. Balbo à E. Mounier, du 29 août 1947, réf. citées.

51. C'est Pellegrini qui attire l'attention de Mounier sur ce groupe (lettre d'A. Pellegrini à E. Mounier, du 21 septembre 1947, réf. citées).

52. Emmanuel Mounier, «Lignes de force d'un personnalisme italien », Esprit, n 141 , janvier 1948, p. 14-23. 
d'un « témoignage ${ }^{53}$ qui est le rapport que les catholiques communistes turinois ont présenté au congrès d'Esprit organisé en France en juillet $1947^{54}$, puis d'un texte d'Elio Vittorini sur les rapports entre politique et culture et d'un article du critique André Bazin sur l'école de cinéma néo-réaliste ${ }^{55}$.

La réception des réalités culturelles et politiques italiennes à Esprit à été possible grâce aux liens étroits noués par Mounier dans des réseaux d'intellectuels transalpins depuis 1946. Les deux parties exercent tour à tour la fonction de médiateur culturel : l'équipe d'Esprit dont le rôle s'apparente, envers les groupes italiens, à celui d'un «tuteur idéologique », et les réseaux d'intellectuels italiens qui informent Mounier et les siens et les introduisent dans l'univers culturel italien. À travers la reconstitution de ces liens et réseaux apparaissent en filigrane les grandes lignes du contenu des articles sur la péninsule publiés dans Esprit au début de 1948 et, au-delà, les contours de la connaissance de l'Italie que la revue diffuse à son lectorat.

La réintroduction en France des réalités italiennes passe par des médiateurs culturels et des réseaux intellectuels. Outre leur fonction médiatrice, ils ont également une influence sur les contours de la connaissance qu'ils transmettent, en effectuant des choix, en interprétant les faits par le prisme de leur idéologie. Trois facteurs, renvoyant aux rôles des émigrés politiques transalpins, de la sphère éditoriale et des groupes d'intellectuels liés à la revue Esprit, sont à l'origine de l'attrait pour l'Italie des médiateurs culturels et de la constitution des réseaux intellectuels.

Si l'impact du nouveau discours à propos de l'Italie sur l'imaginaire des hommes politiques et des diplomates et, partant, sur les rapports politiques franco-italiens, demeure difficile à évaluer, il ne fait guère de doutes que sa portée soit restée faible sur l'opinion publique française. La réception des films néo-réalistes et, plus encore, des œuvres littéraires réalistes ne concerne guère le grand public qui accorde ses préférences au Petit monde de don Camillo de Giovanni Guareschi ${ }^{56}$ et au film qui en est tiré et tend à délaisser les œuvres d'un Rossellini ou d'un Vittorini. L'engouement pour la production réaliste italienne, au lendemain de la guerre, se limite aux intellectuels de gauche qui, inversement, ignorent ou stigmatisent le livre de Guareschi et son adaptation au cinéma ${ }^{57}$. Les clercs les plus italophiles appartiennent

53. «Témoignage », Esprit, op. cit., p. 23-33.

54. Rapport mis en forme par l'un des membres du centre personnaliste turinois, Ettore Sobrero, qui a participé au congrès d'Esprit (lettres d'E. Sobrero à E. Mounier, du 27 août 1947, du 25 et du 27 septembre 1947, réf. citées). 55. Elio Vittorini, « Politique et culture (Lettre à Togliatti) », Esprit, op. cit., p. 34-57; André Bazin, « Le réalisme cinématographique et l'école italienne de la Libération », Esprit, op. cit., p. 58-83.

56. Avec 798000 exemplaires, c'est le livre le plus vendu en France entre 1945 et 1955.

57. Le film est une coproduction franco-italienne réalisée par Julien Duvivier. Voici les réflexions qu'il inspire à Xavier Tilliette dans la revue Positif : «Un film en cet automne 1952, draine la clientèle, engorge les salles, étire 
aux milieux communistes situés à la périphérie du parti et à la mouvance de la gauche marxiste indépendante du PCF. Si certains de ces groupes sont culturellement influents et disposent d'un fort pouvoir de légitimation, ils constituent toutefois des cercles assez restreints.

Une ultime observation a trait aux facteurs expliquant leur intérêt pour l'Italie ; outre ceux évoqués précédemment, un dernier élément renvoie à la tendance de ces intellectuels à aller chercher outre-monts des modèles politico-idéologiques qui n'existent pas en France (le parti d'action et les idées du socialisme libéral, le courant des catholiques communistes) ou qui ne revêtent pas les mêmes caractéristiques (un PCI jugé plus souple et plus intellectuel que son homologue français, et doté, grâce aux réflexions de Gramsci, d’un patrimoine idéologique dont est dépourvu le PCF). La valorisation de ces modèles transalpins leur permet de conforter leurs propres positions dans la cléricature française. Pareillement, l'enthousiasme pour la littérature et le cinéma réalistes est aussi alimenté par la carence, en France, de semblables courants après 1945 .

\footnotetext{
les queues aux portes des cinémas dominicaux, crève les plafonds des recettes ! De mémoire de Charlot, on n'avait pas vu un tel triomphe. Tous les gens du petit monde du cinéma, financiers, fabricants, négociants, détaillants se frottent les paumes [...]. Tandis que les critiques et les vrais amis du cinéma remâchent leur amertume, car ce succès, patent, inscrit en gros chiffres sur les colonnes de recettes, est irrémédiable. Il consacre le divorce à très long terme entre la critique et le public, l'inanité de celle-là, le grégarisme de celui-ci » (Xavier Tilliette, « Feux sur Don Camillo. Mon curé chez les producteurs », Positif, n 4, automne 1952, p. 38).
} 